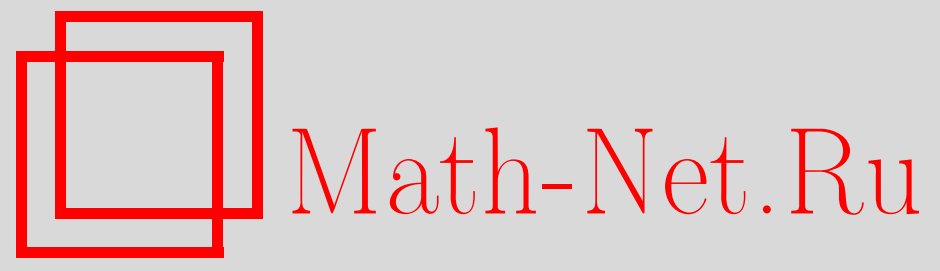

Д. Ю. Черухин, О сложности унитарных преобразований, Дискрет. матем., 2003, том 15, выпуск 4, 113-118

DOI: https://doi.org/10.4213/dm219

Использование Общероссийского математического портала Math-Net.Ru подразумевает, что вы прочитали и согласны с пользовательским соглашением http: //www. mathnet.ru/rus/agreement

Параметры загрузки:

IP : 3.93 .64 .190

26 апреля 2023 г., 17:14:39

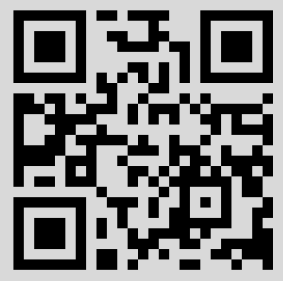




\title{
О сложности унитарных преобразований
}

\author{
○) 2003 г. Д. Ю. Черухин
}

В работе предложен метод получения нижних оценок сложности неветвящихся программ, элементарными операциями в которых являются унитарные преобразования над двумя комплексными числами. Метод позволяет получать оценки вида $\Omega(n \log n)$ для унитарных операторов $\mathbf{C}^{n} \rightarrow \mathbf{C}^{n}$, в частности, для преобразований Фурье и Уолша. При $n=2^{k}$ найдены точные значения сложности последних.

Работа выполнена при поддержке Российского фонда фундаментальных исследований по Программе поддержки ведущих научных школ, проект 00-15-96103.

Напомнім, что комплекснозначная матрица $M$ размера $n \times n$ называется унитарной, если обратная матрица к ней совпадает с сопряженной матрицей $M^{*}$, полученной из $M$ транспонированием и комплексным сопряжением коэффициентов. Группу унитарных преобразований $\mathbf{C}^{n} \rightarrow \mathbf{C}^{n}$ обозначим через $U\left(\mathbf{C}^{n}\right)$, преобразования будем отождествлять с их матрицами в стандартном базисе.

Унитарной неветвящейся программой (УНП) назовем последовательность команд $\Pi=\left(K_{1}, \ldots, K_{L}\right)$, имеющих вид

$$
K_{t}=P_{t}\left(a_{t}, b_{t}\right), \quad P_{t} \in \mathcal{U}\left(\mathbf{C}^{2}\right), \quad a_{t}, b_{t} \in \mathbf{Z}_{+}, \quad t=1, \ldots, L .
$$

Пусть имеется неограниченное число комплекснозначных ячеек памяти, занумерованных числами $0,1,2, \ldots$ Тогда действие команды $K_{t}$ состоит в следующем: берутся числа из ячеек с номерами $a_{t}, b_{t}$, с ними производится преобразование $P_{t}$ и результат записывается в те же ячейки. Действие программы П состоит в последовательном применении команд $K_{1}, \ldots, K_{L}$.

Пусть $n$ - натуральное число (размерность исходных данных), $m$ - максимальное из чисел $a_{t}, b_{t}, t=1, \ldots, L$ и числа $n$. Тогда для описания действия программы можно ограничитъся рассмотрением первых $m$ ячеек памяти. Команде $K_{t}$ поставим в соответствие матрицу $M_{t} \in \mathcal{U}\left(\mathbf{C}^{m}\right)$, совпадающую с единичной матрицей всюду, кроме подматрицы, образованной строками и столбцами с номерами $a_{t}, b_{t}$ (всюду в работе будем считать, что нумерация строк и столбцов матрицы ведется с нуля); последняя же подматрица совпадает с матрицей преобразования $P_{t}$. Действие команды $K_{t}$ состоит в умножении вектор-столбца значений ячеек памяти слева на матрицу $M_{t}$, а действие программы Пв умножении на матрицу $M_{L} \ldots M_{1}$.

Пусть $M \in \mathcal{U}\left(\mathbf{C}^{n}\right)$. Скажем, что программа П вычисляет оператор $M$, если матрица $M$ может быть получена из матрицы $M_{L} \ldots M_{1}$ удалением всех столбцов, кроме первых $n$, удалением некоторых $m-n$ строк и перестановкой оставшихся строк. Другими словами, для любого начального состояния памяти, в котором входные данные записаны в первых $n$ ячейках (в остальных ячейках - нули), программа П, примененная к нему, должна 
выдавать вектор значений оператора $M$ на данном входном наборе в некоторых $n$ фиксированных ячейках памяти (неважно, в каком порядке). Число $L$ назовем сложностью программы П. Сложностью оператора $M$ в классе УНП назовем минимальную сложность УНП, вычисляющей оператор $M$. Данную сложность обозначим через $L$ УНП .

Теорема 1. Для любого $M \in \mathcal{U}\left(\mathrm{C}^{n}\right), M=\left(m_{i, j}\right)$, справедливо неравенство

$$
L^{\mathrm{УН \Pi}}(M) \geqslant-\sum_{i, j}\left|m_{i, j}\right|^{2} \log _{2}\left|m_{i, j}\right|,
$$

где $|z|$ - модуль комплексного числа $z$ (при $z=0$ полагаем $\left.|z|^{2} \log _{2}|z|=0\right)$.

Прежде, чем приступить к доказательству теоремы, приведем некоторые ее следствия. Обозначим через $\varphi_{n}$ корень $n$-й степени из единицы, $\varphi_{n}=\exp \{2 \pi i / n\}$. Преобразование Фурье [1] задается матрицей

$$
\Phi_{n}=\frac{1}{\sqrt{n}}\left(\varphi_{n}^{i j}\right), \quad i, j=0,1, \ldots, n-1 .
$$

Пусть $n=2^{k}, 0 \leqslant i, j \leqslant n-1$. Представим числа $i, j$ в двоичной системе счисления:

$$
i=\sum_{l=0}^{k-1} i_{l} 2^{l}, \quad j=\sum_{l=0}^{k-1} j_{l} 2^{l}
$$

и положим

$$
\langle i, j\rangle=\sum_{l=0}^{k-1} i_{l} j_{l} .
$$

Преобразование Уолша имеет матрицу

$$
W_{n}=\frac{1}{\sqrt{n}} A_{n},
$$

где $A_{n}$ - матрица Адамара,

$$
A_{n}=\left((-1)^{\langle i, j\rangle}\right), \quad i, j=0,1, \ldots, n-1 .
$$

Все коэффициенты матриц $\Phi_{n}$ и $W_{n}$ по модулю равны $1 / \sqrt{n}$ (этот множитель введен для того, чтобы преобразование было унитарным).

Следствие 1. Для любого п из области определения

$$
L^{\mathrm{YH \Pi}}\left(\Phi_{n}\right) \geqslant \frac{1}{2} n \log _{2} n, \quad L^{\mathrm{YH \Pi}}\left(W_{n}\right) \geqslant \frac{1}{2} n \log _{2} n .
$$

Известные алгоритмы быстрого преобразования Фурье и Уолша легко моделируются унитарными преобразованиями, что мы и покажем для случая $n=2^{k}$. Пусть $w-$ комплексное число, по модулю равное единице. Введем оператор

$$
U_{w}=\frac{1}{\sqrt{2}}\left(\begin{array}{cc}
1 & 1 \\
w & -w
\end{array}\right)
$$


Обозначим

$$
D_{n}=\operatorname{diag}\left(1, \varphi_{2 n}, \varphi_{2 n}^{2}, \ldots, \varphi_{2 n}^{n-1}\right)
$$

диагональную матрицу размера $n \times n$ с соответствующими элементами на диагонали; через $E_{n}$ будем обозначать единичную матрицу размера $n \times n$, а через $O_{n}$ - нулевую (состоящую из нулей) того же размера.

Легко видеть, что $\Phi_{2}=U_{1}$, поэтому $L^{\text {УНП }}\left(\Phi_{2}\right) \leqslant 1$. Далее, пусть $\Phi_{n}^{\prime}$ - матрица, полученная из $\Phi_{n}$ следующей перестановкой строк: вначале в $\Phi_{n}^{\prime}$ идут строки, имеющие в $\Phi_{n}$ четные номера $0,2, \ldots, n-2$ (с сохранением их порядка), затем - нечетные $1,2, \ldots, n-1$ (также с сохранением порядка). Справедливо представление

$$
\Phi_{2 n}^{\prime}=\frac{1}{\sqrt{2}}\left(\begin{array}{cc}
\Phi_{n} & \Phi_{n} \\
\Phi_{n} D_{n} & -\Phi_{n} D_{n}
\end{array}\right)=\left(\begin{array}{cc}
\Phi_{n} & O_{n} \\
O_{n} & \Phi_{n}
\end{array}\right) \frac{1}{\sqrt{2}}\left(\begin{array}{cc}
E_{n} & E_{n} \\
D_{n} & -D_{n}
\end{array}\right),
$$

в силу которого для вычисления $\Phi_{2 n}$ достаточно применить последовательность команд

$$
U_{1}(0, n), U_{\varphi_{2 n}}(1, n+1), U_{\varphi_{2 n}^{2}}(2, n+2), \ldots, U_{\varphi_{2 n}^{n-1}}(n-1,2 n-1),
$$

а затем вычислить $\Phi_{n}$ для двух наборов ячеек памяти (перестановка строк соответствует изменению номеров ячеек, в которых будет храниться результат, и не влияет на сложность). Таким образом,

$$
L^{\text {УНП }}\left(\Phi_{2 n}\right) \leqslant 2 L^{\text {УНП }}\left(\Phi_{n}\right)+n,
$$

откуда по индукции находим, что

$$
L^{\text {УНП }}\left(\Phi_{n}\right) \leqslant \frac{1}{2} n \log _{2} n .
$$

Для матрищы Адамара справедливо представление

$$
A_{2 n}=\left(\begin{array}{cc}
A_{n} & A_{n} \\
A_{n} & -A_{n}
\end{array}\right),
$$

поэтому матрицу преобрзования Уолша можно представить в виде

$$
W_{2 n}=\frac{1}{\sqrt{2}}\left(\begin{array}{cc}
W_{n} & W_{n} \\
W_{n} & -W_{n}
\end{array}\right)=\frac{1}{\sqrt{2}}\left(\begin{array}{cc}
E_{n} & E_{n} \\
E_{n} & -E_{n}
\end{array}\right)\left(\begin{array}{cc}
W_{n} & O_{n} \\
O_{n} & W_{n}
\end{array}\right) .
$$

Мы видим, что для вычисления $W_{2 n}$ нужно два раза вычислить $W_{n}$ и применить команды $U_{1}(0, n), \ldots, U_{1}(n-1,2 n-1)$. Отсюда с учетом тождества $W_{2}=U_{1}$ получаем, что

$$
L^{\mathrm{YH \Pi}}\left(W_{n}\right) \leqslant \frac{1}{2} n \log _{2} n \text {. }
$$

Следствие 2. Если $n=2^{k}$, то

$$
L^{\mathrm{YH \Pi}}\left(\Phi_{n}\right)=L^{\text {УНП }}\left(W_{n}\right)=\frac{1}{2} n \log _{2} n .
$$

Перейдем к доказательству теоремы. Введем обозначения

$$
\begin{aligned}
& \psi(x)=x \log _{2} x, \quad x \geqslant 0 \quad(\psi(0)=0), \\
& \mathscr{E}(z)=-\frac{1}{2} \psi\left(|z|^{2}\right), \quad z \in \mathbf{C} .
\end{aligned}
$$


Заметим, что

$$
\mathscr{E}(z)=-|z|^{2} \log _{2}|z|
$$

Для произвольной комплекснозначной матрицы $M$ (в том числе для вектора) обозначим через $\mathscr{E}(M)$ сумму величин $\mathscr{E}\left(m_{i, j}\right)$ по всем элементам $m_{i, j}$ матрищы $M$.

Предложение 1. Пусть неотрицательные числа $x, y, x^{\prime}, y^{\prime}$ связаны равенством

$$
x+y=x^{\prime}+y^{\prime}
$$

Toгda

$$
\left|\psi(x)+\psi(y)-\psi\left(x^{\prime}\right)-\psi\left(y^{\prime}\right)\right| \leqslant x+y .
$$

Доказательство. В силу возрастания функции $\log _{2} x$

$$
\psi(x)+\psi(y) \leqslant x \log _{2}(x+y)+y \log _{2}(x+y)=\psi(x+y) .
$$

С другой стороны, функция $\psi(x)$ выпукла, так как

$$
\psi^{\prime \prime}(x)=\left(\log _{2} x+1 / \ln 2\right)^{\prime}=\frac{1}{x \ln 2}>0,
$$

поэтому

$$
\begin{aligned}
\psi(x+y) & =\psi\left(\frac{2 x+2 y}{2}\right) \leqslant \frac{1}{2}(\psi(2 x)+\psi(2 y)) \\
& =x \log _{2}(2 x)+y \log _{2}(2 y)=\psi(x)+\psi(y)+x+y .
\end{aligned}
$$

Из (3), (4) и равенства $x+y=x^{\prime}+y^{\prime}$ следует, что

$$
\begin{aligned}
& \psi(x)+\psi(y)-\psi\left(x^{\prime}\right)-\psi\left(y^{\prime}\right)=(\psi(x)+\psi(y)-\psi(x+y)) \\
&+\left(\psi\left(x^{\prime}+y^{\prime}\right)-\psi\left(x^{\prime}\right)-\psi\left(y^{\prime}\right)\right) \leqslant 0+\left(x^{\prime}+y^{\prime}\right)=x+y,
\end{aligned}
$$

и аналогично,

$$
\psi\left(x^{\prime}\right)+\psi\left(y^{\prime}\right)-\psi(x)-\psi(y) \leqslant x+y .
$$

Неравенства (5) и (6) дают в совокупности оценку (2).

Предложение доказано.

Следствие 3. Пусть комплексные числа $z, w, z^{\prime}, w^{\prime}$ связаны равенством

$$
|z|^{2}+|w|^{2}=\left|z^{\prime}\right|^{2}+\left|w^{\prime}\right|^{2}
$$

Тогда

$$
\left|\mathscr{E}(z, w)-\mathscr{E}\left(z^{\prime}, w^{\prime}\right)\right| \leqslant \frac{1}{2}\left(|z|^{2}+|w|^{2}\right) .
$$

Лемма 1. Пусть $M=\left(m_{i, j}\right) \in \mathcal{U}\left(\mathbf{C}^{m}\right), M_{t}$ - матрица, соответствующая команде $P_{t}$ вида (1). Тогда

$$
\left|\mathscr{E}\left(M_{t} M\right)-\mathscr{E}(M)\right| \leqslant 1 .
$$


Доказательство. Умножение на матрицу $M_{t}$ состоит в преобразовании $a_{t}$-й и $b_{t}$-й строк исходной матрицы; остальные строки остаются неизменными. Обозначим через $\left(z_{0}, \ldots, z_{m-1}\right)$ и $\left(w_{0}, \ldots, w_{m-1}\right)$, соответственно, $a_{t}$-ю и $b_{t}$-ю строки матрицы $M$, а через $\left(z_{0}^{\prime}, \ldots, z_{m-1}^{\prime}\right)$ и $\left(w_{0}^{\prime}, \ldots, w_{m-1}^{\prime}\right)-$ соответствующие строки матрицы $M_{t} M$. Тогда

$$
\mathscr{E}\left(M_{t} M\right)-\mathscr{E}(M)=\sum_{j=0}^{m-1}\left(\mathscr{E}\left(z_{j}, w_{j}\right)-\mathscr{E}\left(z_{j}^{\prime}, w_{j}^{\prime}\right)\right)
$$

Матрицы $M$ и $M_{t}$ унитарны, поэтому $M_{t} M$ унитарна. Сумма квадратов модулей чисел в любой строке и любом столбце унитарной матрицы равна единице, действительно, из равенства $M M^{*}=E_{m}$ следует, что

$$
1=\sum_{l} m_{i, l} \bar{m}_{i, l}=\sum_{l}\left|m_{i . l}\right|^{2}
$$

а из равенства $M^{*} M=E_{m}$ - аналогичное утверждение для столбцов. В частности,

$$
\sum_{j=0}^{m-1}\left|z_{j}\right|^{2}=\sum_{j=0}^{m-1}\left|w_{j}\right|^{2}=1
$$

Кроме того, в силу совпадения $j$-х столбцов матриц $M$ и $M_{t} M$ всюду, кроме $a_{t}$-й и $b_{t}$-й позиций, справедливы равенства

$$
\left|z_{j}\right|^{2}+\left|w_{j}\right|^{2}=\left|z_{j}^{\prime}\right|^{2}+\left|w_{j}^{\prime}\right|^{2}, \quad j=0,1, \ldots, m-1
$$

Наконец, из (7)-(9) и следствия 3 получаем, что

$$
\begin{aligned}
\left|\mathscr{E}\left(M_{t} M\right)-\mathscr{E}(M)\right| & \leqslant \sum_{j=0}^{m-1}\left|\mathscr{E}\left(z_{j}, w_{j}\right)-\mathscr{E}\left(z_{j}^{\prime}, w_{j}^{\prime}\right)\right| \\
& \leqslant \frac{1}{2} \sum_{j=0}^{m-1}\left(\left|z_{j}\right|^{2}+\left|w_{j}\right|^{2}\right)=\frac{1}{2}(1+1)=1 .
\end{aligned}
$$

Лемма доказана.

Доказательство теоремы 1. Рассмотрим программу П вида (1), вычисляющую оператор $M$ и имеющую при этом минимальную сложность. Введем обозначение

$$
M_{\leqslant t}=M_{t} M_{t-1} \ldots M_{1} E_{m}, \quad t=0,1, \ldots L .
$$

Заметим, что для любой унитарной матрицы $M$ выполнено неравенство $\mathscr{E}(M) \geqslant 0$, причем $\mathscr{E}\left(E_{m}\right)=0$ (действительно, все элементы унитарной матрицы по модулю не превосходят единицы, а $\psi(x) \leqslant 0$ при $x \in[0,1]$; кроме того, $\psi(0)=\psi(1)=0)$. Применяя $L$ раз лемму 1 , получаем, что

$$
\mathscr{E}\left(M_{\leqslant L}\right)=\left|\mathscr{E}\left(M_{\leqslant L}\right)-\mathscr{E}\left(M_{\leqslant 0}\right)\right| \leqslant \sum_{t=1}^{L}\left|\mathscr{E}\left(M_{t} M_{\leqslant t-1}\right)-\mathscr{E}\left(M_{\leqslant t-1}\right)\right| \leqslant L .
$$


Матрица $M$ получена из некоторой подматрицы матрицы $M \leqslant L$ перестановкой строк, следовательно, $\mathscr{E}(M) \leqslant \mathscr{E}\left(M_{\leqslant}\right)$. Окончательно получаем, что

$$
L^{\mathrm{YH \Pi}}(M)=L \geqslant \mathscr{E}(M \leqslant L) \geqslant \mathscr{E}(M)
$$

Теорема доказана.

Заметим, что в основе квантовых вычислений [2] (более сильных, чем булевы), лежат унитарные преобразования, однако доказанная теорема дословно не переносится на квантовый случай из-за параллелизма последнего (на каждом шаге вычисления изменяются значения всех коэффищиентов).

Автор благодарен О. Б. Лупанову за внимание к работе.

\section{Список литературы}

1. Сэвидж Дж. Э., Сложсиость вычислений. Факториал, Москва, 1998.

2. Стин Э., Квантовые вычисления. НИЦ «Регулярная и хаотическая динамика», Ижевск, 2000.

Статья поступила 10.07.2003. 